\title{
Hamiltonian Formulation for Nonlinear Sloshing in Layered Two Immiscible Fluids*
}

\author{
Kensuke HARA** and Hiroki TAKAHARA*** \\ ** Department of Mechanical Engineering, Aoyama Gakuin University \\ 5-10-1 Fuchinobe, Sagamihara-shi, Kanagawa, 229-8558, Japan \\ E-mail: hara@me.aoyama.ac.jp \\ *** Department of Mechanical Sciences and Engineering, Tokyo Institute of Technology \\ 2-12-1 Ookayama, Meguro-ku, Tokyo, 152-8550, Japan
}

\begin{abstract}
This paper deals with a nonlinear surface and interface sloshing in layered two immiscible fluids of different desities. Scientific interest in surface and interface sloshing includes the need to quantify allowance loads on separators or agitators in chemical plants and so on. We propose the analytical method for nonlinear sloshing in this problem by using Hamiltonian formulation. In theoretical analysis, the governing equations and canonical form (Hamiltonian equations) of a system of two fluids with a dynamic free surface and interface are given by applying Hamilton's principle. Moreover, the nonlinear ordinary differential system which governs liquid surface and interfacial wave motions is derived by using Dirichlet-Neumann operators and the generalized Fourier series expansion. Solving these ordinary differential system yields the time histories and the transitions of surface and interfacial wave motions in a rectangular tank. The validly of the theoretical analysis is verified through the experiments.
\end{abstract}

Key words : Sloshing, Nonlinear Vibration, Method of Vibration Analysis, Hamiltonian, Canonical Conjugate Variables, Dirichlet-Neumann Operator

\section{Introduction}

Surface and interface sloshing occur in a tank containing two immiscible fluids of different densities. Scientific interest in this problem includes the need to quantify overflow and allowance loads on separators or agitators in chemical plants, and so on.

Up to this time, the problems of surface and interface sloshing have been reported in Refs. (1) - (4). Handa and Tajima ${ }^{(1)}$ studied dispersion relations and amplitude-frequency responses of a two-dimensional model. Tang ${ }^{(2)}$ studied time histories of surface wave elevation and pressure of circular cylindrical tank. These studies are focused on a linear problem of the layered fluid system. On the other hand, Craig et al..$^{(3)}$ studied a Hamiltonian formulation of the internal wave problem on ocean engineering. They conducted a derivation of the approximating equations which can be applicable to the shallow water problem and a investigation for the effect of the nonlinearity on dispersion relations.

In the previous study, we gave canonical conjugate variables and Hamiltonian equations for this problem by a variational formulation ${ }^{(4)}$. Then, the experimental and theoretical analyses are conducted to investigate the effect of surface tension on the natural frequencies and the linearized problems for this system (vibration modes and time histories). However, we couldn't conduct the nonlinear analysis for time histories of surface and interfacial elevations. In this study, we derive the Hamiltonian equations which involve the effect of nonlinearity. Transitions of surface and interfacial elevations and time histories of surface elevations are calculated by solving these equations. Moreover, the validly of the analytical results are verified through the experiments. 


\section{Nomenclature}

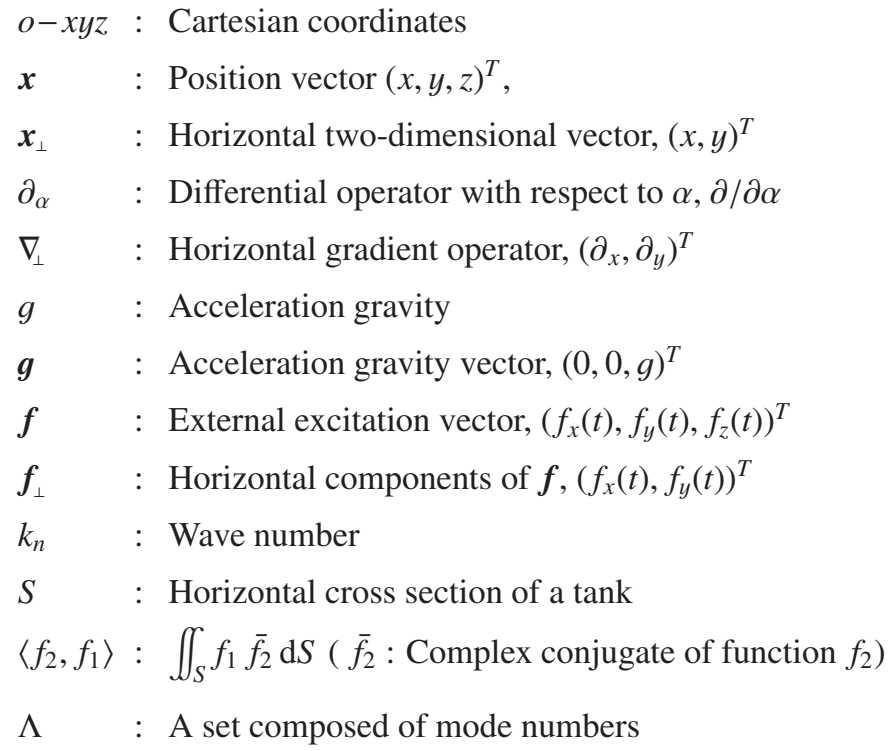

\section{Formulation of the Problem}

\subsection{Analytical Model and Non-dimensional Parameters}

Figure 1 shows the analytical model of this problem. We consider two immiscible fluids with constant densities $\rho^{(1)}$ and $\rho^{(2)}$, respectively. The surface tension has negligible effects on this system [See Ref. (4)]. The moving coordinate system $o-x y z$ is fixed on the tank which has a uniform cross section $S$. The origin $o$ of $x y z$ is located on the undisturbed interface. The tank is subjected to the horizontal and vertical excitations $\boldsymbol{f}=\left(f_{x}(t), f_{y}(t), f_{z}(t)\right)^{T}$. The surface $z=\eta^{(1)}(x, y, t)=\eta^{(1)}\left(\boldsymbol{x}_{\perp}, t\right)$ (the interface) separates the two fluids and the surface $z=\eta^{(2)}\left(\boldsymbol{x}_{\perp}, t\right)$ (the free surface) separates the upper fluid from the air, supposed to be a constant atmosphereic pressure $P_{0}=0$. This system is in a stable configuration in that $\rho^{(1)}>\rho^{(2)}$. Moreover, the density ratio of two fluids is defined as follows:

$$
\rho:=\frac{\rho^{(2)}}{\rho^{(1)}} .
$$

In such configuration, the fluid is assumed to be incompressible, inviscid, and irrotational. Therefore, we can define velocity potentials in the lower and upper fluid domains. The velocity potentials are expressed as

$$
\left.\begin{array}{l}
\Phi^{(1)}(\boldsymbol{x}, t) \text { in the lower fluid domain } V^{(1)}, \\
\Phi^{(2)}(\boldsymbol{x}, t) \text { in the upper fluid domain } V^{(2)} .
\end{array}\right\}
$$

These velocity potentials satisfy

$$
\nabla^{2} \Phi^{(i)}=0 \quad(i=1,2),
$$

where $\nabla=\left(\partial_{x}, \partial_{y}, \partial_{z}\right)^{T}$. The fluid velocity components normal to the fixed boundaries are equal to zero. Hence, the velocity components on the wall satisfy

$$
\left.\boldsymbol{n}_{w} \cdot \nabla \Phi^{(i)}\right|_{\text {wall }}=0 \quad(i=1,2),
$$

where $\boldsymbol{n}_{w}$ is the unit exterior normal vector to the wall.

In order to obtain the spatially dimensionless equations, we introduce the following quantities:

$$
\left.\begin{array}{l}
\tilde{x}=x / \ell, \tilde{y}=y / \ell, \tilde{z}=z / \ell, \tilde{h}^{(i)}=h^{(i)} / \ell, \tilde{\eta}^{(i)}=\eta^{(i)} / \ell, \\
\tilde{\boldsymbol{f}}=\boldsymbol{f} / \ell, \tilde{\boldsymbol{g}}=\boldsymbol{g} / \ell, \tilde{\Phi}^{(i)}=\Phi^{(i)} / \ell^{2}
\end{array}\right\}
$$


where $\ell$ is the characteristic length. Using the these spatially dimensionless quantities and dropping the tildes, we express the governing equations of this system in the following analysis.

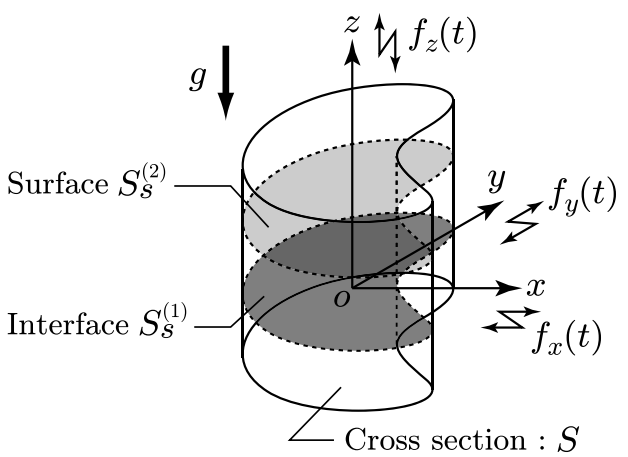

(a) Analytical domain

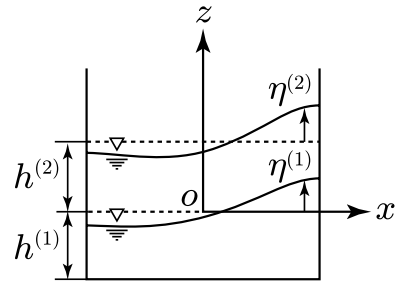

(b) Side view

Fig. 1 Analytical model

\subsection{Hamiltonian of This System}

In the previous study, we proposed a Hamiltonian formulation and a Hamiltonian functional for the layered fluid system ${ }^{(4)}$. The Hamiltonian functional $\mathcal{H}$ of this system is defined as the sum of kinetic energy and potential energy ${ }^{(4)}$, namely,

$$
\begin{aligned}
& \mathcal{H}=\mathcal{T}+\mathcal{U} \\
& \mathcal{T}=: \iint_{S} \frac{1}{2}\left[\begin{array}{l}
\xi^{(1)} \\
\xi^{(2)}
\end{array}\right]^{T}\left[\begin{array}{l}
E^{(11)} E^{(12)} \\
E^{(21)} E^{(22)}
\end{array}\right]\left[\begin{array}{c}
\xi^{(1)} \\
\xi^{(2)}
\end{array}\right] \mathrm{d} S, \\
& \mathcal{U}=: \iint_{S}\left\{\frac{1}{2}\left(g+\ddot{f_{z}}\right)\left[(1-\rho) \eta^{(1)^{2}}+\rho \eta^{(2)^{2}}\right]\right. \\
&\left.+\ddot{\boldsymbol{f}}_{\perp} \cdot \boldsymbol{x}_{\perp}\left[(1-\rho) \eta^{(1)}+\rho \eta^{(2)}\right]\right\} \mathrm{d} S .
\end{aligned}
$$

where $\mathcal{T}$ and $\mathcal{U}$ represent a kinetic and a potential energy, respectively. $\xi^{(i)}(i=1,2)$ are canonical momenta on surface and interface ${ }^{(4)}$. Those are defined as

$$
\left.\begin{array}{rl}
\xi^{(1)} & :=\phi^{(1)}-\rho \phi^{(2)}, \\
\xi^{(2)} & :=\rho \phi^{(3)} .
\end{array}\right\}
$$

The variables $\phi^{(i)}(i=1,2,3)$ represent boundary values of velocity potential on surface and interface. These variables are defined as

$$
\left.\begin{array}{rl}
\phi^{(1)} & :=\left.\Phi^{(1)}\right|_{z=\eta^{(1)}}, \\
\phi^{(2)} & :=\left.\Phi^{(2)}\right|_{z=\eta^{(1)}}, \\
\phi^{(3)} & :=\left.\Phi^{(2)}\right|_{z=h^{(2)}+\eta^{(2)}}
\end{array}\right\}
$$

The variables $\eta^{(i)}$ and $\xi^{(i)}$ are called canonical variables. $E^{(i j)}$ represent the nonlinear differential operators. The operators $E^{(i j)}$ are given by

$$
\left.\begin{array}{l}
E^{(11)}=G^{(11)}\left(G^{(11)}+\rho G^{(l)}\right)^{-1} G^{(l)}, \\
E^{(12)}=-G^{(l)}\left(G^{(11)}+\rho G^{(l)}\right)^{-1} G^{(12)}, \\
E^{(21)}=-G^{(21)}\left(G^{(11)}+\rho G^{(l)}\right)^{-1} G^{(l)}, \\
E^{(22)}=-\frac{1}{\rho}\left[G^{(22)}+G^{(21)}\left(G^{(11)}+\rho G^{(l)}\right)^{-1} G^{(12)}\right] .
\end{array}\right\}
$$

where $G^{(l)}$ and $G^{(i j)}$ represent the Dirichlet-Neumann operators for two fluid domains ${ }^{(4)}$. The expressions for $G^{(l)}$ and $G^{(i j)}$ are given in the section 3.4. 


\subsection{Hamiltonian Equations (Canonical Equations)}

The Hamiltonian of this system is given by Eqs.(6), (7), and (8). By applying the variational principle to this expression for the Hamiltonian ${ }^{(4)}$, Hamiltonian equations of this system take the form

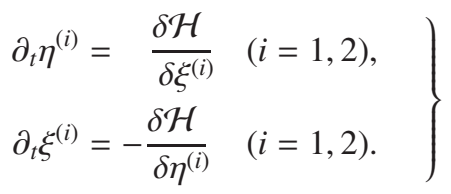

where $\delta \mathcal{H} / \delta f$ is the functional derivative of $\mathcal{H}$ with respect to $f$ [See Ref. (4)].

The next procedure is to express the Hamiltonian equations (12) in the modal coordinate. It is equivalent to applying a generalized Fourier series expansion to the canonical equations Eq. (12). The generalized Foureier series is an expansion of a function in terms of an infinite sum of arbitrary orthonormal functions. In this study, we use the orthonormal function which satisfy the condition (3) and (4).

The velocity potentials $\Phi^{(i)}(i=1,2)$ which satisfy Eqs. (3) and (4) are expressed as

$$
\begin{aligned}
& \Phi^{(1)}=\sum_{n \in \Lambda} \Phi_{n}^{(1)}(t) \psi_{n}(x, y) \frac{\cosh \left[k_{n}\left(z+h^{(1)}\right)\right]}{\cosh \left(k_{n} h^{(1)}\right)}, \\
& \Phi^{(2)}=\sum_{n \in \Lambda} \Phi_{n}^{(2)}(t) \psi_{n}(x, y)\left(e^{k_{n} z}+c_{n} e^{-k_{n} z}\right),
\end{aligned}
$$

where $c_{n}$ are constants and $\Lambda$ is a set composed of mode numbers. Moreover, the constants $k_{n}$ are the wave number and $\psi_{n}$ are the orthonormal functions, which satisfy

$$
\begin{aligned}
& \left(\nabla_{\perp}^{2}+k_{n}^{2}\right) \psi_{n}=0, \\
& \left.\boldsymbol{n}_{w} \cdot \nabla \psi_{n}\right|_{\text {wall }}=0 \quad(i=1,2) .
\end{aligned}
$$

From Eqs.(9), (13) and (14), the generalized Fourier series of canonical variables can be written as

$$
\left.\begin{array}{ll}
\eta^{(i)}=\sum_{n \in \Lambda} \eta_{n}^{(i)}(t) \psi_{n}(x, y) & (i=1,2), \\
\xi^{(i)}=\sum_{n \in \Lambda} \xi_{n}^{(i)}(t) \psi_{n}(x, y) & (i=1,2) .
\end{array}\right\}
$$

Using the definition of functional derivative ${ }^{(4)}$ and the generalized Fourier series (17), the Hamiltonian equation (12) can be rewritten as

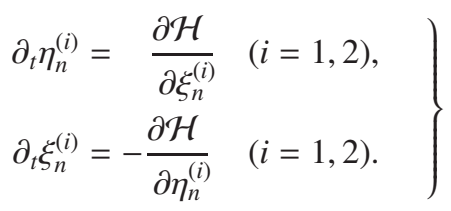

\subsection{Dirichlet-Neumann Operators}

In order to express the operators $E^{(i j)}$ in explicit forms, we derive the expressions of the Dirichlet-Neumann operators $G^{(l)}$ and $G^{(i j)}$. These operators are defined as

$$
G^{(l)} \phi^{(1)}:=\left.\left(1+\left|\nabla_{\perp} \eta^{(1)}\right|^{2}\right)^{\frac{1}{2}} \boldsymbol{n}_{s}^{(1)} \cdot \nabla \Phi^{(1)}\right|_{z=\eta^{(1)}},
$$

which is the Dirichlet-Neumann operator for the lower fluid domain ${ }^{(4)}$, and

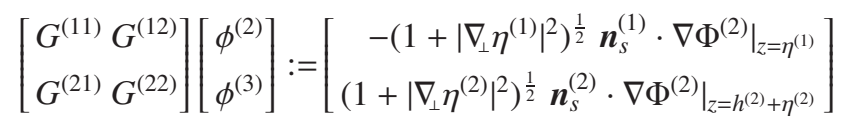

which is the Dirichlet-Neumann operator for the upper fluid domain ${ }^{(4)}$. The vectors $\boldsymbol{n}_{s}^{(1)}$ and $\boldsymbol{n}_{s}^{(2)}$ are unit exterior normal vectors to the lower fluid domain along the free interface and the 
upper fluid domain along the free surface. The operators $G^{(l)}$ and $G^{(i j)}$ are nonlinear differential operators. The specific expressions of $G^{(l)}$ and $G^{(i j)}$ can be expressed as the following power series expansions in terms of $\eta^{(i)}(i=1,2)$.

$$
\begin{aligned}
G^{(l)}\left(\eta^{(1)}\right) & :=\sum_{m_{1}=0}^{\infty} G_{m_{1}}^{(l)}, \\
G^{(i j)}\left(\eta^{(1)}, \eta^{(2)}\right) & :=\sum_{m_{1}=0}^{\infty} \sum_{m_{2}=0}^{\infty} G_{m_{1}, m_{2}}^{(i j)},
\end{aligned}
$$

where $m_{1}$ and $m_{2}$ are the order of the power series of $\eta^{(1)}$ and $\eta^{(2)}$, respectively. Then, the Taylor series expansion of Eq. (13) at $z=0$ take the form

$$
\Phi^{(1)}=\sum_{n=0}^{\infty} \sum_{r=0}^{\infty} \Phi_{n}^{(1)} \psi_{n}\left[\frac{\left(k_{n} z\right)^{2 r}}{2 r !}+\tanh \left(k_{n} h^{(1)}\right) \frac{\left(k_{n} z\right)^{2 r+1}}{(2 r+1) !}\right] .
$$

The Taylor series expansions of Eq. (14) at $z=0, h^{(2)}$ are expressed as

$$
\begin{aligned}
& \Phi^{(2)}=\sum_{n=0}^{\infty} \sum_{r=0}^{\infty} \Phi_{n}^{(2)} \psi_{n} \frac{\left(k_{n} z\right)^{r}}{r !}\left[1-c_{n}(-1)^{r}\right], \\
& \Phi^{(2)}=\sum_{n=0}^{\infty} \sum_{r=0}^{\infty} \Phi_{n}^{(2)} \psi_{n} \frac{\left[k_{n}\left(z-h^{(2)}\right)\right]^{r}}{r !}\left[e^{k_{n} h^{(2)}}-c_{n}(-1)^{r} e^{-k_{n} h^{(2)}}\right] .
\end{aligned}
$$

Substituting Eqs. (10), (21) and (23) into Eq. (19), one has

$$
\begin{aligned}
& G^{(l)} \sum_{r=0}^{\infty}\left[\frac{\left(k_{n} \eta^{(1)}\right)^{2 r}}{2 r !}+\tanh \left(k_{n} h^{(1)}\right) \frac{\left(k_{n} \eta^{(1)}\right)^{2 r+1}}{(2 r+1) !}\right] \psi_{n} \\
& =\sum_{r=0}^{\infty} \frac{\left(k_{n} \eta^{(1)}\right)^{2 r}}{2 r !}\left[k_{n} \tanh \left(k_{n} h^{(1)}\right)-\left(\nabla_{\perp} \eta^{(1)} \cdot \nabla_{\perp}\right)\right] \psi_{n} \\
& +\sum_{r=0}^{\infty} \frac{\left(k_{n} \eta^{(1)}\right)^{2 r+1}}{(2 r+1) !}\left[k_{n}-\tanh \left(k_{n} h^{(1)}\right)\left(\nabla_{\perp} \eta^{(1)} \cdot \nabla_{\perp}\right)\right] \psi_{n} .
\end{aligned}
$$

Reading the zero- and first-order terms of the Taylor expansions in $\eta^{(1)}$ from Eqs. (21) and (26), we find

$$
\begin{aligned}
G_{0}^{(l)} f\left(\boldsymbol{x}_{\perp}, t\right) & =G_{0}^{(l)} \sum_{n \in \Lambda}\left\langle\psi_{n}, f\right\rangle \psi_{n} \\
& =\sum_{n \in \Lambda} k_{n} \tanh \left(k_{n} h^{(1)}\right)\left\langle\psi_{n}, f\right\rangle \psi_{n}, \\
G_{1}^{(l)} f\left(\boldsymbol{x}_{\perp}, t\right) & =\left(-\nabla_{\perp} \cdot \eta^{(1)} \nabla_{\perp}-G_{0}^{(l)} \eta^{(1)} G_{0}^{(l)}\right) f,
\end{aligned}
$$

where $f$ is the arbitrary function.

There is an analogous procedure for derivations of the Dirichlet-Neumann operator $G^{(i j)}$ for upper domain. Substituting Eqs. (22), (24) and (25) into Eq. (20), one has

$$
\begin{aligned}
& G^{(11)} \sum_{r=0}^{\infty} \frac{\left(k_{n} \eta^{(1)}\right)^{r}}{r !}\left[1-c_{n}(-1)^{r}\right] \psi_{n} \\
& +G^{(12)} \sum_{r=0}^{\infty} \frac{\left(k_{n} \eta^{(2)}\right)^{r}}{r !}\left[e^{k_{n} h^{(2)}}-c_{n}(-1)^{r} e^{-k_{n} h^{(2)}}\right] \psi_{n} \\
& =\sum_{r=0}^{\infty} \frac{\left(k_{n} \eta^{(1)}\right)^{r}}{r !}\left\{c_{n}(-1)^{r}\left(k_{n}+\nabla_{\perp} \eta^{(1)} \cdot \nabla_{\perp}\right)\right. \\
& \left.+\left(-k_{n}+\nabla_{\perp} \eta^{(1)} \cdot \nabla_{\perp}\right)\right\} \psi_{n} \\
& G^{(21)} \sum_{r=0}^{\infty} \frac{\left(k_{n} \eta^{(1)}\right)^{r}}{r !}\left[1-c_{n}(-1)^{r}\right] \psi_{n} \\
& +G^{(22)} \sum_{r=0}^{\infty} \frac{\left(k_{n} \eta^{(2)}\right)^{r}}{r !}\left[e^{k_{n} h^{(2)}}-c_{n}(-1)^{r} e^{-k_{n} h^{(2)}}\right] \psi_{n}
\end{aligned}
$$




$$
\begin{aligned}
=\sum_{r=0}^{\infty} \frac{\left(k_{n} \eta^{(2)}\right)^{r}}{r !}\left\{c_{n}(-1)^{r+1} e^{-k_{n} h^{(2)}}\left[k_{n}+\nabla_{\perp} \eta^{(1)} \cdot \nabla_{\perp}\right]\right. \\
\left.+e^{k_{n} h^{(2)}}\left[k_{n}-\nabla_{\perp} \eta^{(1)} \cdot \nabla_{\perp}\right]\right\} \psi_{n} .
\end{aligned}
$$

The relations Eqs. (29) and (30) can be solved for the Taylor coefficients of the DirichletNeumann operator $G^{(i j)}$ as a double power series in $\eta^{(1)}$ and $\eta^{(2)}$.

From comparison in the relations Eqs. (29) and (30), one finds that the constant (zeroorder) terms in the Taylor expansions are

$$
G_{0,0}^{(i j)} f=\left\{\begin{array}{c}
\sum_{n \in \Lambda} k_{n} \operatorname{coth}\left(k_{n} h^{(2)}\right)\left\langle\psi_{n}, f\right\rangle \psi_{n} \quad(i=j), \\
-\sum_{n \in \Lambda} k_{n} \operatorname{csch}\left(k_{n} h^{(2)}\right)\left\langle\psi_{n}, f\right\rangle \psi_{n}(i \neq j) .
\end{array}\right.
$$

where csch is cosech. Reading the first-order terms of the Taylor expansions in $\eta^{(1)}$ and $\eta^{(2)}$ from Eqs. (29) and (30), we find

$$
\left.\begin{array}{l}
G_{1,0}^{(11)} f=\left(\nabla_{\perp} \cdot \eta^{(1)} \nabla_{\perp}+G_{0,0}^{(11)} \eta^{(1)} G_{0,0}^{(11)}\right) f \\
G_{1,0}^{(12)} f=G_{0,0}^{(11)} \eta^{(1)} G_{0,0}^{(12)} f \\
G_{1,0}^{(21)} f=G_{0,0}^{(12)} \eta^{(1)} G_{0,0}^{(11)} f \\
G_{1,0}^{(22)} f=G_{0,0}^{(12)} \eta^{(1)} G_{0,0}^{(12)} f \\
G_{0,1}^{(11)} f=-G_{0,0}^{(12)} \eta^{(2)} G_{0,0}^{(12)} f \\
G_{0,1}^{(12)} f=-G_{0,0}^{(12)} \eta^{(2)} G_{0,0}^{(11)} f \\
G_{0,1}^{(21)} f=-G_{0,0}^{(11)} \eta^{(2)} G_{0,0}^{(12)} f \\
G_{0,1}^{(22)} f=-\left(\nabla_{\perp} \cdot \eta^{(2)} \nabla_{\perp}+G_{0,0}^{(11)} \eta^{(2)} G_{0,0}^{(11)}\right) f
\end{array}\right\}
$$

\subsection{Lower-order Hamiltonian}

The Hamiltonian functional $\mathcal{H}$ is expressed as a Taylor series in the canonical variables $\left(\eta^{(1)}, \eta^{(2)}, \xi^{(1)}, \xi^{(2)}\right)$. The approximating equations are obtained by retaining a finite number of terms in the Taylor expansion in canonical variables of the Hamiltonian.

The Taylor expansion of Hamiltonian $\mathcal{H}$ in powers of canonical variables is given by

$$
\mathcal{H}=\mathcal{H}_{f}+\sum_{n \geq 2}^{\infty} \mathcal{H}_{n},
$$

where $n$ is the sum of powers of canonical variables $\left(\eta^{(1)}, \eta^{(2)}, \xi^{(1)}, \xi^{(2)}\right)$ and $\mathcal{H}_{f}$ is the effect of external force in Hamiltonian $\mathcal{H}$. In this paper, we consider the quadratic nonlinearity for the canonical variables. Using the expressions (6), (11), (21) and (22) and retaining terms of up to third order in the Taylor expansion of Hamiltonian $\mathcal{H}$ [Eq. (34)], the resulting Hamiltonian can be expressed as

$$
\begin{aligned}
\mathcal{H}= & \mathcal{H}_{f}+\mathcal{H}_{2}+\mathcal{H}_{3} \\
\mathcal{H}_{f}= & \sum_{n_{1} \in \Lambda}\left\langle\psi_{n_{1}}, \boldsymbol{x}_{\perp}\right\rangle \cdot \ddot{\boldsymbol{f}}_{\perp}\left[(1-\rho) \eta_{n_{1}}^{(1)}+\rho \eta_{n_{1}}^{(2)}\right] \\
& +\frac{1}{2} \sum_{n_{1} \in \Lambda} \ddot{f}_{z}\left[(1-\rho) \eta_{n_{1}}^{(1)^{2}}+\rho \eta_{n_{1}}^{(2)^{2}}\right] \\
\mathcal{H}_{2}= & \frac{1}{2} \sum_{n_{1} \in \Lambda}\left[\Gamma_{n_{1}}^{(21)} \xi_{n_{1}}^{(1)^{2}}+\Gamma_{n_{1}}^{(22)} \xi_{n_{1}}^{(1)} \xi_{n_{1}}^{(2)}+\Gamma_{n_{1}}^{(23)} \xi_{n_{1}}^{(2)^{2}}\right. \\
& \left.+(1-\rho) g \eta_{n_{1}}^{(1)^{2}}+\rho g \eta_{n_{1}}^{(2)^{2}}\right] \\
\mathcal{H}_{3}= & \frac{1}{2} \sum_{n_{1}, n_{2}, m_{1} \in \Lambda} \sum_{n_{1}, n_{2}, m_{1}} \xi_{n_{1}}^{(1)} \xi_{n_{2}}^{(1)} \eta_{m_{1}}^{(1)}
\end{aligned}
$$




$$
\begin{aligned}
& +\Gamma_{n_{1}, n_{2}, m_{1}}^{(32)} \xi_{n_{1}}^{(1)} \xi_{n_{2}}^{(1)} \eta_{m_{1}}^{(2)}+2 \Gamma_{n_{1}, n_{2}, m_{1}}^{(33)} \xi_{n_{1}}^{(1)} \xi_{n_{2}}^{(2)} \eta_{m_{1}}^{(1)} \\
& +2 \Gamma_{n_{1}, n_{2}, m_{1}}^{(34)} \xi_{n_{1}}^{(1)} \xi_{n_{2}}^{(2)} \eta_{m_{1}}^{(2)}+\Gamma_{n_{1}, n_{2}, m_{1}}^{(35)} \xi_{n_{1}}^{(2)} \xi_{n_{2}}^{(2)} \eta_{m_{1}}^{(1)} \\
& \left.+\Gamma_{n_{1}, n_{2}, m_{1}}^{(36)} \xi_{n_{1}}^{(2)} \xi_{n_{2}}^{(2)} \eta_{m_{1}}^{(2)}\right]
\end{aligned}
$$

where $\Gamma^{(\cdot)}$ are constants with time. The specific expressions for $\Gamma^{(\cdot)}$ are given by Appendix A. Substituting Eq. (35) into (18), we can obtain nonlinear time history responses for surface and interfacial elevations.

\subsection{Example for Two-Dimensional Model}

Figure 2 shows the two-dimensional rectangular model. Tank has a length of $2 L$. It is subjected to a horizontal excitation $f_{x}=f_{0} \sin \omega t$. The origin of the moving coordinate system $o-x z$ is located at the center of the undisturbed free interface. In this case, the wave number $k_{n}$ and the orthonormal function $\psi_{n}$ which satisfy Eqs.(15) and (16) are given by

$$
\begin{aligned}
\psi_{n}(x) & =X_{n}(x), \\
k_{n} & =\frac{\pi}{2} n, \\
X_{n}(x) & = \begin{cases}\frac{1}{\sqrt{2}} & (n=0), \\
\cos \left[\frac{n}{2} \pi(x+1)\right] & (n \neq 0) .\end{cases}
\end{aligned}
$$

\section{Experiment}

\subsection{Specifications of Model Tank and Fluids}

Tables 1 and 2 represent the parameters of the fluids and the tank used in the analyses and experiments, respectively. The test fluids are water and flourinert ${ }^{\mathrm{TM}} \mathrm{FC}-3283$ (Sumitono $3 \mathrm{M} \mathrm{Ltd}$.). In this study, we conducted the calculations and experiments in the cases of the shallow water (Case 1) and deep water (Case 2). In the previous study, we showed that this system has two coupled resonance modes which have same wave number ${ }^{(4)}$. In this study, we describe the vibration modes that the surface elevations are larger than interfacial elevations as "surface mode" and the other vibration modes as "interfacial modes". The excitation angular frequencies are nearly the 1 st surface and interfacial resonance modes. The natural frequencies of these cases are shown in table 3 .

\subsection{Experimental Setup}

Figure 3 shows a schematic diagram of the experimental setup. The tank is mounted on a shake table. The personal computer controls the input signal (sine wave) to a hydraulic

Table 1 The properties of fluid $\left(25^{\circ} \mathrm{C}\right)$

\begin{tabular}{ccc}
\hline Fluid & Water & Fluorinert \\
\hline Density $\left[\mathrm{kg} / \mathrm{m}^{3}\right]$ & 997 & 1830 \\
\hline
\end{tabular}

Table 2 The parameters of experiment and calculation

\begin{tabular}{lcc}
\hline & Case 1 & Case 2 \\
\hline External excitation $f_{x}(t)$ & \multicolumn{2}{c}{$f_{0} \sin \omega t$} \\
Length of tank $2 L$ & {$[\mathrm{~mm}]$} & \multicolumn{2}{c}{378} \\
Height of lower fluid $h^{(1)}[\mathrm{mm}]$ & 28.35 & 75.6 \\
Height of upper fluid $h^{(2)}[\mathrm{mm}]$ & 28.35 & 75.6 \\
Considered modes & \multicolumn{2}{c}{$\psi_{1}, \cdots, \psi_{11}$} \\
Observation point $(x, y)$ & {$[\mathrm{mm}]$} & $(164,0)$ \\
\hline
\end{tabular}


Table 3 The natural frequencies $[\mathrm{rad} / \mathrm{s}]$

\begin{tabular}{ccccc}
\hline & \multicolumn{2}{c}{ Case 1 } & \multicolumn{2}{c}{ Case 2 } \\
$n$ & Interfacial & Surface & Interfacial & Surface \\
\hline 1 & 2.23 & 5.63 & 3.50 & 8.10 \\
2 & 4.37 & 10.5 & 6.27 & 12.6 \\
3 & 6.36 & 14.4 & 8.24 & 15.6 \\
\hline
\end{tabular}

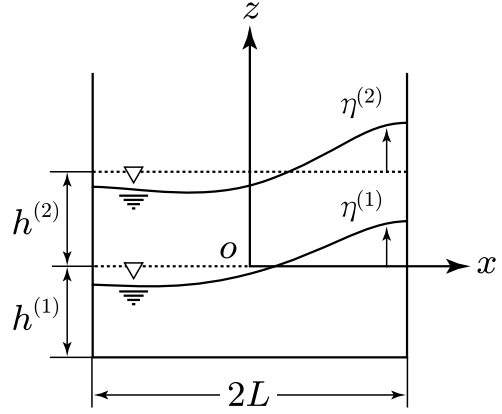

Fig. 2 Model tank

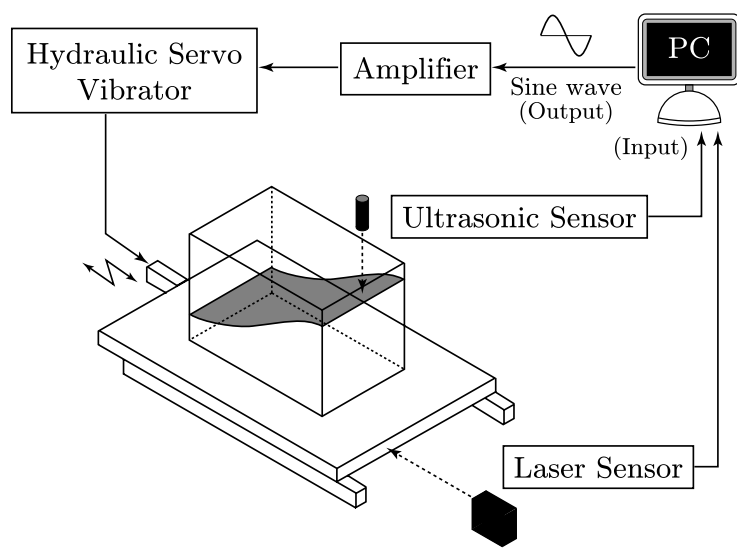

Fig. 3 Experimental setup

servo vibrator. The shake table is excited by the hydraulic servo vibrator. The liquid surface elevation is measured by a ultrasonic sensor. Moreover, the displacement of shake table is measured by a laser sensor.

\section{Results}

\subsection{Transitions of Surface and Interfacial Elevations}

Analytical and experimental results for transitions of the surface and interfacial elevations are shown in Figs. 4, 5 and 6. The upper and lower figures show the analytical and experimental results, respectively. These results indicate that the analytical and experimental results are in reasonable agreement with each other. Figure 4 represents the result of the 1 st surface mode resonance in the case of shallow water. This result indicates that a transition of the surface elevation became a traveling wave. The surface elevation shows a large displacement when the wave reflects on the wall (at $t=10.50[\mathrm{~s}]$ ). This is similar to the case of a sloshing in a single layer fluid.

Figures 5 and 6 show the results of the 1st surface and interfacial modes resonances in the case of deep water, respectively. These results indicate that transitions of the surface and interface elevations became nearly sinusoidal waves. Therefore, the nonlinear effects are weak in the case of deep water. The 3rd order interfacial mode is slightly observed in Fig. 6. This is because the natural frequency of the 1st order surface mode is nearly equal to the natural frequency of the 3 rd order interfacial mode (See in table 3 ).

\subsection{Time Histories of Surface Elevations}

Figures 7 and 8 represent time histories of surface elevation of the 1st surface mode resonances in the case 1 and case 2, respectively. The measurement point is a neighborhood of the side wall $(x=164[\mathrm{~mm}])$. These results show the good agreements with each other. Figure 7 shows the analytical and experimental results of surface elevations in the shallow water case (Case 1). This result indicates that the positive displacement is larger than the negative displacement. It is attributed to the superpositions of high-frequency components by nonlinearity.

Figure 8 shows the analytical and experimental result of surface elevations in the deep water case (Case 2). The positive displacements are slightly larger than negative displacements 

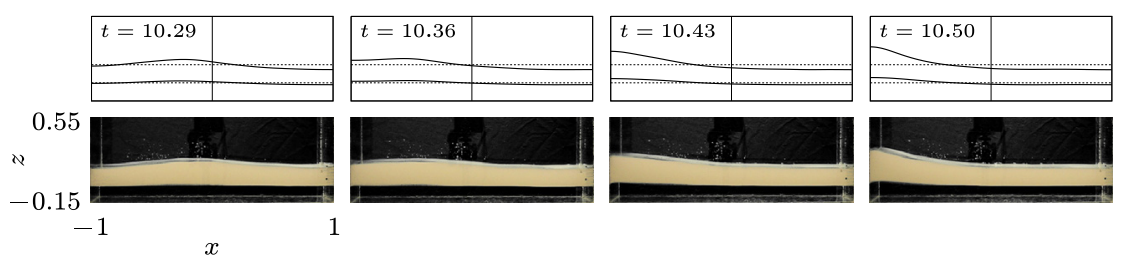

Fig. 4 Transitions of wave elevations (Case 1, $\left.f_{0}=1.28[\mathrm{~mm}], \omega=5.53[\mathrm{rad} / \mathrm{s}]\right)$
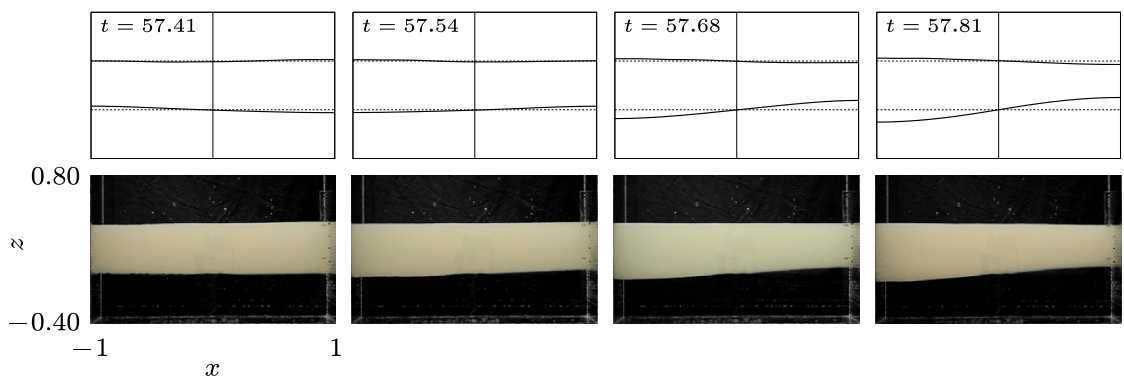

Fig. 5 Transitions of wave elevations (Case 2, $f_{0}=2.63[\mathrm{~mm}], \omega=3.45[\mathrm{rad} / \mathrm{s}]$ )
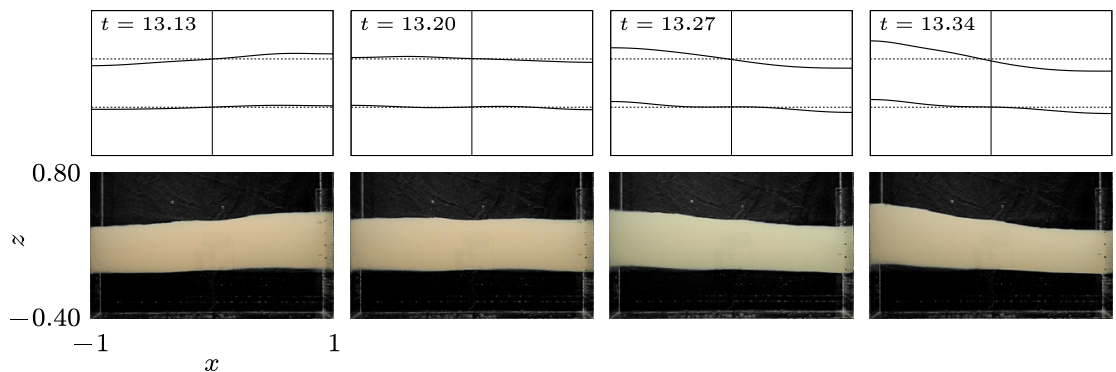

Fig. 6 Transitions of wave elevations (Case 2, $f_{0}=0.60[\mathrm{~mm}], \omega=8.35[\mathrm{rad} / \mathrm{s}]$ )

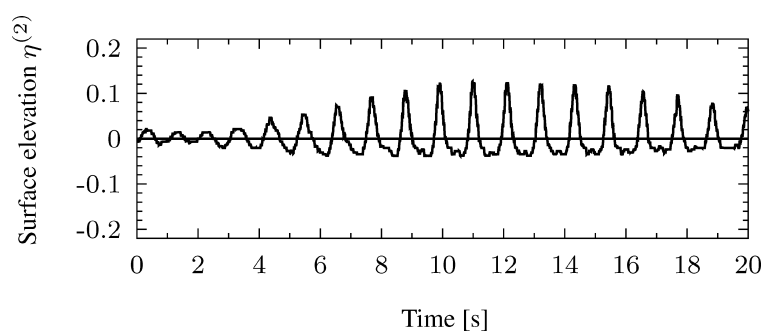

(a) Experimental result

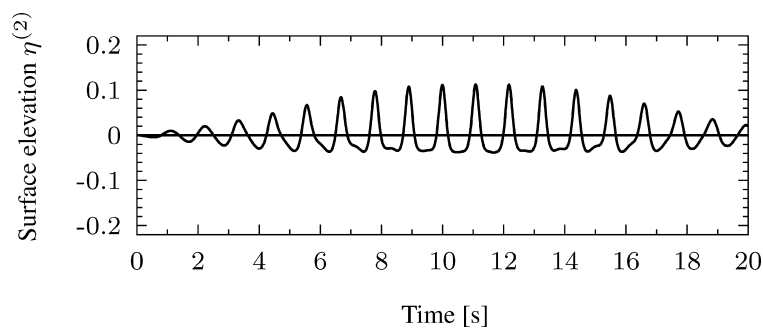

(b) Analytical result

Fig. 7 Time histories of free surface elevation (Case 1, $f_{0}=1.05[\mathrm{~mm}], \omega=5.53[\mathrm{rad} / \mathrm{s}]$ )

in the analytical and experimental results. However, these time histories are nearly sinusoidal. We can find that the nonlinear effects are weak in this case.

\section{Conclusions}

In this paper, we derived the Hamiltonian equations which govern the nonlinear sloshing in layered two immiscible fluids. Moreover, the caluclation results are validated by the experimental results. The results are as follows. 


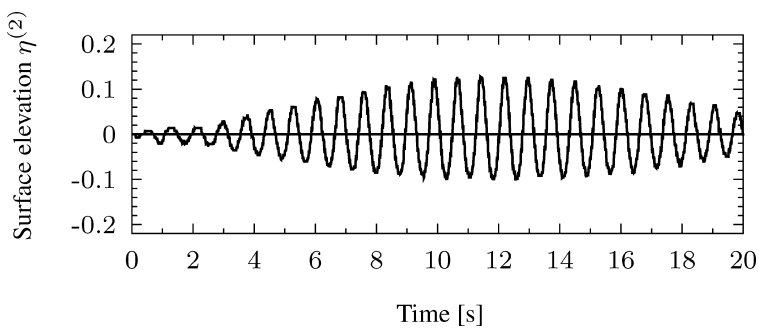

(a) Experimental result

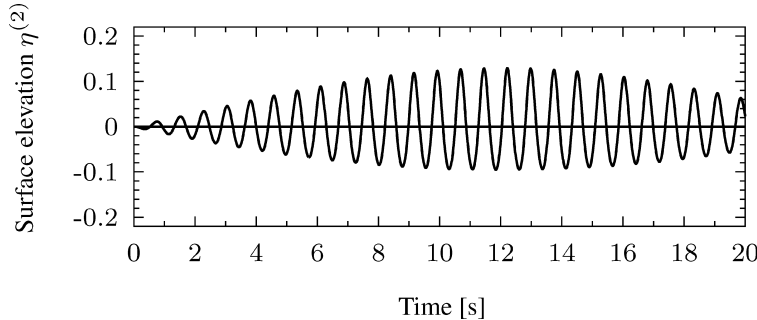

(b) Analytical result

Fig. 8 Time histories of free surface elevation (Case 2, $f_{0}=0.55[\mathrm{~mm}], \omega=8.35[\mathrm{rad} / \mathrm{s}]$ )

( 1 ) The governing equations of nonlinear sloshing in layered two immiscibles fluids are derived by using the Hamiltonian formulation and Dirichlet-Neumann operators.

(2) By considering the 3rd order Hamiltonian, the analytical and experimental results are in good agreement with each other. Therefore, The effect of the 3rd order Hamiltonian (the quadratic order nonlinearity) is important for the calculations.

(3) The surface and interfacial modes resonate at the same time when the difference between these natural frequencies are small.

\section{A. Coefficients in Hamiltonian Functional}

$$
\begin{aligned}
\mathrm{T}_{n}^{(i)} & =\tanh \left(k_{n} h^{(i)}\right) \quad(i=1,2) \\
\mathrm{C}_{n}^{(i)} & =\cosh \left(k_{n} h^{(i)}\right) \quad(i=1,2) \\
\mathrm{S}_{n}^{(i)} & =\sinh \left(k_{n} h^{(i)}\right) \quad(i=1,2) \\
\Gamma_{n}^{(21)} & =\frac{k_{n} \mathrm{~T}_{n}^{(1)}}{1+\rho \mathrm{T}_{n}^{(1)} \mathrm{T}_{n}^{(2)}} \\
\Gamma_{n}^{(22)} & =\frac{2}{\mathrm{C}_{n}^{(2)}} \Gamma_{n}^{(21)} \\
\Gamma_{n}^{(23)} & =\frac{1}{\rho}\left(\frac{\mathrm{T}_{n}^{(2)}}{\mathrm{T}_{n}^{(1)}}+\rho\right) \Gamma_{n}^{(21)} \\
\Gamma_{a, b, c}^{(31)} & =\Gamma_{a}^{(21)} \Gamma_{b}^{(21)}\left[(\rho-1)\left\langle\psi_{b}, \psi_{a} \psi_{c}\right\rangle\right. \\
+ & \frac{\left.1-\rho \mathrm{T}_{a}^{(1)} \mathrm{T}_{b}^{(1)} \mathrm{T}_{a}^{(2)} \mathrm{T}_{b}^{(2)}\left\langle\psi_{c}, \nabla_{\perp} \psi_{a} \nabla_{\perp} \psi_{b}\right\rangle\right]}{k_{a} k_{b} \mathrm{~T}_{a}^{(1)} \mathrm{T}_{b}^{(1)}} \\
\Gamma_{a, b, c}^{(32)} & =-\frac{\rho}{4} \Gamma_{a}^{(22)} \Gamma_{b}^{(22)}\left\langle\psi_{b}, \psi_{a} \psi_{c}\right\rangle \\
\Gamma_{a, b, c}^{(33)} & =-\frac{1}{2} \Gamma_{a}^{(21)} \Gamma_{b}^{(22)}\left[(\rho-1)\left\langle\psi_{b}, \psi_{a} \psi_{c}\right\rangle+\frac{1+\mathrm{T}_{a}^{(1)} \mathrm{T}_{a}^{(2)}}{k_{a} k_{b} \mathrm{~T}_{a}^{(1)} \mathrm{T}_{b}^{(1)}}\left\langle\psi_{c}, \nabla_{\perp} \psi_{a} \nabla_{\perp} \psi_{b}\right\rangle\right] \\
\Gamma_{a, b, c}^{(34)} & =-\frac{\rho}{2} \Gamma_{a}^{(22)} \Gamma_{b}^{(23)}\left\langle\psi_{b}, \psi_{a} \psi_{c}\right\rangle \\
\Gamma_{a, b, c}^{(35)} & =\frac{\rho-1}{4} \Gamma_{a}^{(22)} \Gamma_{b}^{(22)}\left[\frac{\left\langle\psi_{c}, \nabla_{\perp} \psi_{a} \nabla_{\perp} \psi_{b}\right\rangle}{\left.\rho k_{a} k_{b} \mathrm{~T}_{a}^{(1)} \mathrm{T}_{b}^{(1)}+\left\langle\psi_{b}, \psi_{a} \psi_{c}\right\rangle\right]}\right. \\
\Gamma_{a, b, c}^{(36)} & =\frac{1}{\rho}\left\langle\psi_{c}, \nabla_{\perp} \psi_{a} \nabla_{\perp} \psi_{b}\right\rangle-\frac{1}{\rho} \frac{k_{a} k_{b}\left\langle\psi_{c}, \psi_{a} \psi_{b}\right\rangle}{\mathrm{T}_{a}^{(2)} \mathrm{T}_{b}^{(2)}} \\
&
\end{aligned}
$$




$$
+\left(\frac{k_{b} \mathrm{~T}_{b}^{(1)} \Gamma_{a}^{(22)}}{\mathrm{S}_{a}^{(2)} \mathrm{T}_{b}^{(2)}}+\frac{k_{a} \mathrm{~T}_{a}^{(1)} \Gamma_{b}^{(22)}}{\mathrm{S}_{b}^{(2)} \mathrm{T}_{a}^{(2)}}-\frac{\Gamma_{a}^{(22)} \Gamma_{b}^{(22)}}{2 \mathrm{~S}_{a}^{(2)} \mathrm{S}_{b}^{(2)}}\right) \frac{\left\langle\psi_{b}, \psi_{a} \psi_{c}\right\rangle}{2 \rho \mathrm{T}_{a}^{(1)} \mathrm{T}_{b}^{(1)}}
$$

\section{References}

( 1 ) Handa, K., and Tajima, K., Sloshing of Two Superposed Liquid Layers in Rectangular Tank, Transactions of the Japan Society of Mechanical Engineers, Series B, Vol.45, No.398 (1979), pp.1450-1457.

( 2 ) Tang, Y., Sloshing Displacements in a Tank Containing Two Liquids, Proceedings of the 1993 ASME PVP Conference, Vol.258 (1993-7), pp.179-184.

( 3 ) Craig, W., Guyenne, P. and Kalisch, H., Hamiltonian Long-Wave Expansions for Free Surfaces and Interfaces, Communications on Pure and Applied Mathematics, Vol.LVIII (2005), pp.1587-1641.

( 4 ) Hara, K. and Hiroki, T., Hamiltonian Formulation of Surface and Interface Sloshing in a Tank Containing Two Immiscible Fluids, Journal of System Design and Dynamics, Vol.2, No.1 (2008), pp.299-310. 